\title{
Espacio audiovisual argentino: claroscuros de un presente exitoso y de un futuro incierto
}

\author{
Santiago Marino \\ Universidad Nacional de Quilmes
}

\section{Resumen}

Recibido: 6 de noviembre de 2013. Aceptado: 29 de noviembre de 2013.

El sistema argentino de medios de radiodifusión se ha caracterizado históricamente por su estructura privada y comercial, con acciones estatales que tendieron, en la mayor parte de los procesos, a beneficiar esta lógica. Desde el advenimiento de la democracia, la ley de radiodifusión fue modificada para facilitar la creación de conglomerados, permitir el ingreso de capitales extranjeros y reforzar el sistema basado en la búsqueda de lucro. El resultado muestra que la concentración de propiedad de los medios creció de manera constante y cada nuevo gobierno reforzó la estructura heredada.

Ahora bien, si se piensa en espacio audiovisual ampliado y se incluye el sector cinematográfico, el recorrido se vuelve más complejo, dada la diversidad en las políticas implementadas para cada espacio. De un lado, la regulación y la acción pública con rasgos negativos para la democratización. Del otro, una política de estado a largo plazo con beneficios para la producción nacional e interrogantes sobre el futuro.

El presente trabajo realiza un recorrido por las políticas públicas de comunicación del sector audiovisual (TV y cine) en la Argentina. En primer lugar, se propone una breve sistematización de la política de cine. En segundo lugar, un análisis de la etapa abierta en 2008 y que continúa hasta la actualidad y ha puesto a los medios de comunicación en el centro del debate. Por último, se plantea un cierre con una serie de interrogantes respecto de las encrucijadas que atraviesa el sector en la dinámica actual.

Palabras clave: políticas públicas, medios audiovisuales, cine, radiodifusión, legislación, Argentina.

\section{Argentine audiovisual space: lights and shadows of a successful present and an uncertain future}

\section{Abstract}

The Argentine radio broadcasting system has historically been characterized by its private and commercial structure, with State actions that, in most processes, have tended to benefit this logic. Since the coming of democracy, the broadcasting law was modified to facilitate 
the creation of media conglomerates, allow the entry of foreign capital, and reinforce a system based on the search for profit. The outcome shows that the concentration of media property grew at a constant rate and that each new government reinforced the inherited structure.

Now, if one considers an expanded audiovisual space, which includes the film sector, then the journey becomes more complex, given the diversity of policies implemented in each space. On the one hand, we find regulation and public action with negative aspects for democratization. On the other, a long-term State policy with benefits for national production and questions about the future.

This paper outlines a tour of public communication policies in the Argentine audiovisual sector (film and TV). Firstly, it proposes a brief systematization of film policy. Secondly, it carries out an analysis of the period that began in 2008 and continues to this day, which has placed communication media at the center of debate. Lastly, a conclusion is offered, with a series of questions regarding the dilemmas faced by the sector in the current dynamic.

Keywords: public policies, audiovisual media, film, broadcasting, legislation, Argentina.

\section{Espaço audiovisual argentino: claro-escuros de um presente de sucesso e de um futuro incerto}

O sistema argentino de radiodifusão tem se caraterizado historicamente pela sua estrutura privada e comercial, com ações estatais que tenderam, na maioria dos processos, a beneficiar essa lógica. Desde a chegada da democracia, a lei de radiodifusão foi alterada para facilitar a criação de conglomerados, permitindo o ingresso de capitais estrangeiros e reforçando o sistema baseado na busca do lucro. O resultado mostra que a concentração de propriedade dos meios de comunicação cresceu de forma constante e cada novo governo reforçou a estrutura herdada.

Pois bem, se pensamos sobre o espaço audiovisual expandido e se incluímos o setor cinematográfico, o percorrido se torna mais complexo, dada a diversidade nas políticas implementadas para cada espaço. De um lado, a regulação e a ação pública com traços negativos para a democratização. Do outro lado, uma política de estado de longo prazo com benefícios para a produção nacional e perguntas sobre o futuro.

O presente trabalho realiza um percorrido pelas políticas públicas de comunicação do setor audiovisual (TV e cinema) na Argentina. Em primeiro lugar, propõe-se uma breve sistematização da política do cinema. Em segundo lugar, uma análise da etapa aberta em 2008 que continua até a atualidade e tem colocado os meios de comunicação no centro do debate. Por último, expõe-se, para concluir, uma série de perguntas a respeito das encruzilhadas que atravessa o setor na dinâmica atual.

Palavras chave: políticas públicas, meios audiovisuais, cinema, radiodifusão, legislação, Argentina. 


\section{Introducción}

En la cultura de la sociedad de masas, los medios de comunicación son una parte importante del espacio público propiamente dicho, en cuanto instituciones complejas por las cuales transitan discursos, polifonías, sentidos y donde además se configuran las identidades culturales. Dado que el desarrollo capitalista se caracteriza por mercantilizar las distintas actividades sociales, la industrialización de la comunicación en particular se aborda aquí desde una visión crítica de la economía política de la información y la comunicación, en directa relación con la tradición de los estudios de las políticas de comunicación y los marcos regulatorios de los distintos sectores de las industrias culturales.

Los fenómenos que involucran procesos comunicacionales analizados desde perspectivas abarcadoras y críticas dan cuenta de los modos en que se articulan la cultura, la comunicación, la economía y la política, en una relación interdisciplinaria que intenta ampliar la visión, desarrollar estudios comparativos sobre sectores diferentes durante el mismo período temporal y brindar elementos para comprender recorridos complejos. Al plantear la temática y los abordajes de esta investigación se sostiene la convicción de que los lineamientos culturales, políticos y económicos están directamente involucrados, y pensarlos en conjunto permite distinguir elementos que se vuelven sistemáticos en el proceso de construcción de una sociedad.

Este trabajo parte de la necesidad de comprender el modo en que el Estado administra y regula el sistema de medios, como así también su interacción con el mercado. La intención es aportar elementos para comprender los mecanismos de la implementación de políticas públicas con rasgos diferentes en sectores muy importantes de la cultura y la comunicación argentina. El análisis comparativo de estos casos permitirá dar cuenta de acciones con características divergentes aplicadas en dos áreas del espacio audiovisual en el marco de un mismo modelo de Estado. El tema en estudio es el de las políticas públicas de comunicación. Implica el abordaje comparativo de las intervenciones estatales en la televisión por cable y el cine en la Argentina. El recorrido se propone dar cuenta del modo en que funciona cada sector y las causas y consecuencias de las políticas en su estructura.

El sistema argentino de medios de radiodifusión se ha caracterizado históricamente por su estructura privada y comercial, con acciones estatales que tendieron, en la mayor parte de los procesos, a beneficiar esta lógica. Desde el advenimiento de la democracia la ley de radiodifusión generada en los años de la dictadura fue 
modificada para crear conglomerados, permitir el ingreso de capitales extranjeros y reforzar el sistema basado en la búsqueda de lucro. El resultado muestra que la concentración de propiedad de los medios creció de manera constante y que cada nuevo gobierno reforzó la estructura heredada.

Ahora bien, si se piensa en un espacio audiovisual ampliado y se incluye al sector cinematográfico, el recorrido se vuelve más complejo dada la diversidad en las políticas implementadas para cada grupo. De un lado, la regulación y la acción pública con rasgos negativos para la democratización. Del otro, una política de Estado a largo plazo con beneficios para la producción nacional e interrogantes sobre el futuro.

El presente trabajo presenta un recorrido por las políticas públicas de comunicación del sector audiovisual (TV y cine) en la Argentina. En primer lugar, se propone una breve sistematización de la política de cine. En segundo lugar, un análisis de la etapa abierta en 2008 y que continúa hasta la actualidad y ha puesto a los medios de comunicación en el centro del debate. Por último, se plantea un cierre con una serie de interrogantes respecto de las encrucijadas que atraviesa el sector en la dinámica actual.

\section{El cine}

En la Argentina, la composición del sector cinematográfico posee una peculiaridad: cuenta con una política pública de largo plazo que establece mecanismos de estímulo y protección a la producción local que la distingue del resto del espacio audiovisual y ha sido sostenida más allá de cambios de gobierno y de la gestión estatal. Su legitimidad es amplia y su permanencia parece indiscutible a pesar de lo desparejo de sus resultados, del paso del tiempo y de la necesidad de repensar algunas de sus líneas, con el objetivo de optimizar el uso de sus recursos y ampliar las condiciones de acceso de los ciudadanos a este tipo de contenidos fundamentales en la construcción de identidades culturales nacionales.

Desde la lógica de intervención implementada a partir de la reforma normativa de $1994^{1}$, a contramano del modelo neoliberal imperante en ese momento, la cinematografía es una actividad subsidiada y funciona con un sistema de créditos y subvenciones que resultan indispensables para llevar a cabo las producciones. Ese tipo de ayuda es necesaria porque las inversiones casi nunca se recuperan me-

${ }^{1}$ Ley de Fomento de la Actividad Cinematográfica Nacional, nº 24.377 (19 oct. 1994). 
diante la venta de entradas y otros ingresos ${ }^{2}$. Además, como en el resto del mundo, los grandes estudios norteamericanos arrasan en la taquilla con sus "tanques", motivo por el cual las distribuidoras y las exhibidoras generan mayores ganancias con las películas extranjeras y no invierten en la producción local. La permanencia en el tiempo de la política de cine en nuestro país consolidó un sistema con sus rasgos positivos - como el fomento a la producción y (en menor medida) a la exhibición- y limitaciones notables (la concentración y extranjerización de la propiedad en las instancias de producción y distribución, la escasez de pantallas) que aún no pueden resolverse.

El cine es un medio peculiar tanto por sus rasgos económicos como por su rol en la construcción de relatos que aportan a la constitución de identidades. En países como la Argentina se requiere de políticas públicas que garanticen su existencia, dada su estructura de costos, el tamaño del mercado y el dominio de las producciones extranjeras. La industria local es aparentemente débil pero compone, a su vez, la segunda en idioma español y es una de las diez más importantes de occidente $^{3}$. Muchos de los actores del sector consideran que el cine argentino no es una industria sino más bien una sucesión de proyectos dispares en los que la mayoría tiene una vida de exhibición muy corta e intrascendente. En este esquema, solo unos pocos filmes resultan "elegidos" por el público, superan la cantidad necesaria de espectadores para cubrir los costos de producción y convertirse en éxitos de taquilla (unos 700 mil tickets) y de alguna manera hacer posible la generación del resto (Perelman \& Seivach, 2003, p. 73).

\section{Cuándo, cómo y por qué}

El proceso en que se encuadra el diseño de la política pública de cine en el país abre una etapa novedosa para la cinematografía nacional e incluye una serie de transformaciones socioculturales muy importantes incluso en términos tecnológicos, ya que desde 1991 se dio una importante actualización del equipamiento a partir de la convertibilidad peso/dólar. De todos modos, el contexto para la industria nacional era de gran incertidumbre debido a la crisis que vivía desde finales de la década del 80 y justo entre 1994 y 1995 — cuando el proyecto neoliberal

\footnotetext{
2 Productos de marketing, derechos de antena, entre otros.

3 Luego de España. Véase además: Rovito, P. (2010). "El nuevo paradigma del negocio cinematográfico" (p. 105112). En: Valor y símbolo, dos siglos de industrias culturales en la Argentina. Buenos Aires: Secretaría de Cultura. Sistema de Información Cultural de la Argentina (SINCA).
} 
comenzaba a mostrar sus primeras fisuras en lo social y en lo económico ${ }^{4}$ - el gobierno del entonces presidente Carlos Menem implementó una reforma en el campo cinematográfico cuyos resultados se traducirían en un notable incremento de la actividad productiva (Getino, 2005). Respecto de la gestión estatal, luego de que se sucedieran distintos directores en el Instituto Nacional de Cine y Artes Audiovisuales (INCAA) sin poder resolver la crisis, la reforma de la ley de cine transformaría la realidad definitivamente.

El "espacio cinematográfico argentino" se organizó para demandar una política que defendiera las producciones locales, debido a la precariedad de su situación, a la complejidad de su estructura y al rol de los capitales extranjeros y sus filmes internacionales en el mercado local ${ }^{5}$. La existencia de un ambiente consolidado, integrado por diversos colectivos y actores con un interés común aparece como un elemento clave al momento de comenzar a trabajar con la reforma de la normativa que regula la actividad cinematográfica. Ese rasgo resulta sistemático y perdura a pesar de las diferentes necesidades y demandas hacia adentro del propio espacio.

Con la reforma normativa se habilitó una serie de herramientas para una política de cine que inauguraría una nueva etapa en la actividad cinematográfica y audiovisual nacional. En un estado de crisis casi terminal, la producción local de películas se reactivó fundamentalmente a partir de 1995 con nuevos subsidios derivados del gravamen a los medios electrónicos. Ese momento se conjugó además con la aparición de nuevos técnicos y realizadores, egresados de las escuelas de cine, que suelen ser ubicados - por los contenidos de sus producciones- y definidos como "el nuevo cine argentino" (NCA) ${ }^{6}$. En ese colectivo se integraron producciones de películas de carácter autoral, con estilos y tratamientos variados, incluso con diversidad en sus resultados medidos en éxito de taquilla pero que fundamentalmente establecen una nueva relación con el público y abren mercados antes impensados.

\footnotetext{
4 Con serios perjuicios para el conjunto de las actividades productivas nacionales.

5 Integrado por actores, productores, realizadores y directores entre otros, con una impronta de militancia relevante.

6 Véase además: Algranti, J. M. (2009). "Productores producidos: reflexiones en torno a los circuitos de producción en el nuevo cine argentino”. En: Una década de nuevo cine argentino. Buenos Aires: Ediciones CICCUS.
} 


\section{La política pública de cine}

La actividad cinematográfica en la Argentina estaba regulada por la Ley $17.741^{7}$ y fue reformada mediante las leyes $20.170 / 73,21.505 / 77,24.377 / 94$ y el Decreto $1.536 / 02^{8}$. La norma (con sucesivas reformas y reglamentaciones) establece las condiciones de funcionamiento y fomenta la actividad, crea el organismo de aplicación: el Instituto Nacional de Cine y Artes Audiovisuales (INCAA), define lo que entiende por "película nacional", establece las condiciones para su comercialización y exhibición obligatoria, genera y destina recursos para el crédito y el subsidio a la producción, fomenta las coproducciones, promociona el cine nacional en el exterior y prevé el apoyo y protección al cortometraje. Sus objetivos son el fomento y la regulación de lo que comprende como la producción, la exhibición y la comercialización de películas cinematográficas. Además, incluye distintas resoluciones, como el apoyo a festivales y muestras de cine nacional y de festivales internacionales, junto con los acuerdos de coproducción y codistribución firmados con España, Brasil, Uruguay y Chile, entre otros países. Y se complementó con los cambios aplicados en 2004 a la cuota de pantalla ${ }^{9}$ y la media de continuidad ${ }^{10}$ para los filmes nacionales. El nuevo escenario derivó en un crecimiento importante de la cantidad de películas producidas y estrenadas, como así también en el reconocimiento de la obra de directores argentinos. Pero no podría solucionar dilemas estructurales, como la falta de control para la aplicación de las mejores medidas y las dificultades en las condiciones de estreno y exhibición.

Los principales problemas del cine se materializan en las pobres y precarias condiciones de exhibición de las películas nacionales; en la reducción de la cantidad de pantallas; en su concentración geográfica (en las grandes ciudades y en sus zonas de mayor poder adquisitivo) y en el hecho de que se destine muchísimo dinero (generado por el propio sector pero también por la televisión) a la producción de películas que son vistas por muy pocos. Esta incapacidad del Estado para resolver el dilema de la distribución y la exhibición ${ }^{11}$ se trata de solucionar en la

\footnotetext{
Boletín oficial, 30 de mayo de 1968.

8 Que recuperó la autarquía para el INCAA luego del estallido de la crisis de diciembre de 2001.

9 Es la obligación que tienen los exhibidores de incluir en su programación determinada cantidad de películas nacionales en su cronograma de estrenos.

10 Es la cantidad de espectadores semanales que una película nacional debe alcanzar para que el exhibidor no pueda dejar de exhibirla, es decir "bajarla de cartel". En términos operativos, para defender la industria nacional esa media debe ser algo menor a la media real.

${ }_{11} \mathrm{Y}$ así garantizar mejores condiciones de estrenos para las producciones locales y de acceso para la ciudadanía en tanto que público.
} 
actualidad. En enero de 2012 el INCAA presentó un proyecto que implica reorganizar los lanzamientos de las películas (mediante la creación de un calendario trimestral); reclasificar las salas (en "circuito multipantallas"12 y "circuito ampliado"13 y apoyar con 30.000 pesos $^{14}$ a los filmes que opten por los espacios de menor cantidad de salas para sus estrenos. Está por verse aún su aplicación plena, el cumplimiento de los actores privados y sus resultados.

Por su parte, el modo en que la Ley de Servicios de Comunicación Audiovisual (LSCA) interpeló al cine resultó muy bueno en el marco regulatorio, pero no así en su implementación. Tal como sostiene Esteban Sahores:

Desde la sanción de la LSCA hasta finales de 2012, sostiene el coordinador general de AFSCA (desde abril de 2012), Fernando Pérez, ninguno de los canales presentó la documentación que acredite la compra de los derechos de emisión de películas ni, menos que menos, cumplió con la exhibición de esos estrenos en la pantalla. Según datos aportados por el Sindicato de Cine [...], en los primeros nueve meses de 2012, entre América (cero), Canal 9 (una), Telefé (ocho) y Canal 13 (dos) emitieron apenas once películas argentinas. Entre ellas, una sola inició su rodaje antes de la promulgación de la ley. Se trata del documental sobre Malvinas, 14 de junio, que emitió Canal 9. Pero un informante asegura que el exveterano de Malvinas en persona, productor de la película, la acercó ya terminada y a cambio de nada (Sahores, 2013).

El mismo autor describe que luego de una serie de reuniones entre el secretario de Comercio, Guillermo Moreno, y los ejecutivos de las filiales locales de las cinco majors más poderosas que operan en la Argentina (Sony, Disney, UIP, Warner y Fox), se percibe un nuevo mecanismo de control por parte del capital extranjero del cine en la Argentina, dado que a partir de nuevas alianzas entre los grandes jugadores foráneos y las principales productoras locales, los films generados por la crema de lo industria local apoyados en el star system (ya sea de directores, como Juan José Campanella, o de actores, como Ricardo Darín o Guillermo Francella) comienzan a ser repartidas por las grandes distribuidoras, su impacto en mejores condiciones de estreno es amplio, al igual que su taquilla, pero esto genera un espejismo de desarrollo (ya que son unos pocos productos argentinos los que acceden a dicha condición), mientras que las distribuidoras locales históricamente abocadas a trabajar con material argentino comienzan a perder terreno (Sahores, 2013).

12 Con cuatro pantallas o más.

13 Salas de hasta tres pantallas.

14 Para gastos de distribución y promoción. 


\section{La Argentina de la ley audiovisual}

Luego del estallido de la crisis de 2001 que implicó el final del gobierno de la Alianza, la gestión del entonces presidente Eduardo Duhalde mantuvo en la radiodifusión las condiciones que derivaron en beneficios del sector privado comercial, aunque los mayores efectos de su implementación en el marco regulatorio se materializaron durante la gestión de Néstor Kirchner, culminada en diciembre de 2007.

Por diversas razones (sobre las que aún se discute en profundidad) desde 2008, el gobierno de Cristina Fernández de Kirchner y los grandes grupos de comunicación (fundamentalmente el Grupo Clarín) sostienen un enfrentamiento muy visible cuya cima se produjo a partir de la aprobación de la Ley de Servicios de Comunicación Audiovisual (LSCA) n 26.522/09, que habilitó la transición a un cambio de lógica en el modo de discusión y sanción, establece límites a la concentración de propiedad de los medios y propone nuevas condiciones en la producción de contenidos audiovisuales.

Existen elementos importantes de la nueva ley: se pueden reconocer tres tipos de actores diferentes, uno de los cuales es el no comercial, caracterizado de modo amplio e incluyente, sin caer en el error de hacer lista de tipos de medios no comerciales, que pueden dejar afuera alguno que debería incluir. A su vez, la reserva del 33\% en todas las bandas y en todas las frecuencias es el avance más relevante, sumado a los argumentos que sustentan dicha decisión (su posibilidad de autonomía de este tipo de actor respecto de los poderes económicos y políticos). A esto se puede agregar la posibilidad de otorgar licencias de modo directo a emisores de baja potencia en zonas de no conflicto que podría acelerar el proceso de inclusión de emisores comunitarios.

Además, la inclusión de nuevos límites a la concentración y el avance en la estructura (no aún en su gestión) de los organismos de aplicación y control (AFSCA, Consejo Federal, Comisión Bicameral) son aspectos destacables (Marino, Mastrini \& Becerra, 2010).

Desde su sanción, la Ley de Servicios de Comunicación Audiovisual nunca se aplicó plenamente: en primer lugar, entre 2009 y 2011 primó la reacción de los grandes grupos concentrados para frenar la ley (y la permeabilidad de algunos sectores judiciales); en segundo lugar, la falta de decisión integral del gobierno, y en último término, el rol de la mayoría de los partidos de oposición que tomaron como propia la agenda de los grupos concentrados y deslegitimizaron la ley con sus acciones. De 2011 en adelante los factores se alternaron, primó la reducción 
que el gobierno realizó de la norma al "paquete Clarín", se mantuvo el accionar de sectores judiciales que demoraron las decisiones en torno de las medidas cautelares que suspendieron la aplicación de la ley o de algunos artículos, mientras que la oposición cambió su estrategia pero fue incapaz de generar efectos, como constituir la Bicameral y nombrar sus directores en la AFSCA.

Este último aspecto se resolvió hacia finales de 2012. El Poder Ejecutivo designó como presidente de AFSCA al diputado Martín Sabbatella en reemplazo de Santiago Aragón. Al asumir, el nuevo conductor solicitó la conformación de la Bicameral y la designación de los directores por parte de la segunda y tercera minoría. De este modo, fueron propuestos Marcelo Stubrin (por la Unión Cívica Radical, UCR) - quien fue designado e integra el directorio desde entonces- y Alejandro Pereyra (por el Frente Amplio Progresista, FAP), quien sufrió la impugnación de varias organizaciones y no resultó designado en todo el período hasta 2013.

Durante este periplo y en medio de una disputa que también fue discursiva sucedieron jornadas que parecían definitorias y finalmente no lo fueron. A partir de la asunción de Sabbatella y de su decisión de que la fecha en que vencía la medida cautelar (el 7 de diciembre de 2012) aplicara para el resto de los grupos que deben adecuarse a los nuevos límites, el gobierno comenzó una campaña denominada "7D" (por "7 de diciembre") en la que mediante una serie de spots, carteles, publicidad en vía pública y en el programa Fútbol para todos se divulgaba la idea de que en ese día la ley estaría vigente y el Grupo Clarín debería desprenderse de sus medios.

Esa campaña fue una más de las acciones que se superpusieron como jugadas de una partida de ajedrez, aunque con tres actores intervinientes: el gobierno, el Grupo Clarín y distintos fueros judiciales: la sala 1 en lo Civil y Comercial, el Contencioso Administrativo y la Corte Suprema de Justicia de la Nación (CSJN). En mayo de 2012 la sentencia de la CSJN resolvió que el 7 de diciembre vencía el plazo de 36 meses durante el cual el Grupo Clarín estuvo exceptuado de adecuarse a los nuevos límites a la concentración ${ }^{15}$, ya que había litigado la cuestión en sede judicial. Sin embargo, el jueves 6 de diciembre, la Sala 1 en lo Civil y Comercial extendió la medida cautelar y dejó sin efecto la campaña gubernamental sobre el “7D”. Días más tarde, el 14 de diciembre, el juez en lo Contencioso Administrativo, Horacio Alfonso, falló a favor de la constitucionalidad de los artículos denuncia-

15 El resto de los grupos debería haberlo hecho a partir del 22 de diciembre de 2011. 
dos por Clarín y solicitó que se levantara la medida cautelar nuevamente. El 16 de abril de 2013 los jueces de la sala declararon inconstitucionales los artículos, en un fallo débilmente argumentado y muy criticado por varios especialistas en libertad de expresión, como Damián Loreti y Guillermo Mastrini (Mastrini \& Loreti, 2013). El 14 de junio declaró admisible la apelación del Estado nacional, AFSCA, Cablevisión y Grupo Clarín y elevó la causa a la Corte, que debería resolver sobre el fondo de la cuestión.

En agosto de 2013 la CSJN convocó a audiencias públicas, con la peculiar participación de los amicus curiae que defendieron las posturas de uno y otro de los contendientes y no como amigos del "Tribunal".

Finalmente el proceso judicial se completó. Entre la última semana de octubre y la primera de noviembre de 2013 se conocieron dos novedades clave. En primer lugar, el 29 de octubre la Corte decidió —en un fallo histórico, sólido y muy argumentado ${ }^{16}$ - que la Ley de Servicios de Comunicación Audiovisual es constitucional en todos sus aspectos, definió que el Estado debe imponer límites a la concentración para garantizar la libertad de expresión y que los plazos para la adecuación del Grupo Clarín a los nuevos topes estaban vencidos, por lo que la empresa debía cumplir la ley, al igual que el resto. Esta decisión tiene efectos tanto a corto plazo (sobre la estructura del mercado de medios) como a largo (respecto del modo en que se regula el sistema de medios). Además, la reacción de la firma fue la presentación de un plan de adecuación que implica dividir al grupo en seis unidades, de las cuales las dos más importantes quedarían en manos de los propietarios actuales del grupo y el resto se venderían a personas no vinculadas. Tanto este plan como el de las restantes empresas que aún no fueron aprobados deberán ser evaluados por la autoridad de aplicación con criterios claros y equitativos y de su decisión dependerá parte de la legitimidad de la nueva ley y del futuro del sistema de medios.

Hasta ahora, la aplicación sesgada genera una serie de cuestiones pendientes que se suman a los aspectos que la ley no resolvió. Por un lado, están en espera los aspectos más importantes, tales como garantizar la reserva del espectro, otorgar licencias a operadores sin fines de lucro donde el aire está saturado por emisores comerciales, diseñar el plan técnico de frecuencias para conocer el mapa del espectro, dar cuenta de los resultados del censo realizado en 2010 para saber cuántos emisores existen y cuántas frecuencias hay disponibles. Por otro lado, la demora en

16 Véase: Lacunza \& Marino, 2013. 
la apertura del otorgamiento de licencias a las cooperativas de varias ciudades retrasa el ofrecimiento inmediato de un servicio de televisión por cable con calidad óptima y a precios más competitivos que los operadores comerciales, sobre todo en ciudades donde existe un solo operador y pertenece a un grupo oligopólico.

Por último, pero no menos importante, la elaboración de pliegos específicos para el sector que los interpele como organizaciones sin fines de lucro, que den cuenta de sus vínculos con las comunidades que integran, que avalen y reconozcan su capital social es central, y aún es una demanda sin respuesta.

Varias preguntas muy actuales resultan difíciles de responder. Es complejo imaginar quiénes serán los actores interesados en comprar los medios (radios AM y FM, algún canal de TV abierta en Buenos Aires) que deben vender los grupos que se exceden. Otra cuestión es saber qué pasará el día después (que ya no es el 8 de diciembre, sino alguno de lo que resta de 2013). Esta es la pregunta por la aplicación efectiva de la Ley de Servicios de Comunicación Audiovisual, por el funcionamiento del sistema de medios en un nuevo paradigma regulatorio y por la sustentabilidad de los medios en un escenario de cambio constante. Argentina hoy tiene tres políticas para industrias culturales (la ley de cine, la de TDT y la de medios) con tres agentes estatales diferentes (INCAA, Ministerio de Planificación y AFSCA) que trabajan paralelamente temas que deberían ser pensados y gestionados de modo integral y en relación con el sector privado comercial y sin fines de lucro. En definitiva, una política de Estado.

\section{La ley en la pantalla}

La Ley de Servicios de Comunicación Audiovisual debe ser comprendida como punto de partida hacia la democratización del sistema de comunicación y cultura. No es la etapa final de un proceso sino el comienzo de un camino. Ese recorrido que apenas se ha iniciado debería implicar cambios en la estructura y la composición del sistema de medios. Además estipula una serie de condiciones que, de cumplirse, podrán modificar la oferta cultural audiovisual, los tipos de discurso y los contenidos como apertura hacia la diversidad.

Después de poco más de un año de aplicación, puede verse cómo los principales canales de televisión, señales de cable y radios AM o FM comenzaron a distinguir entre los contenidos formales de sus programas y los anuncios publicitarios, unos y otros separados ahora por placas o audios específicos. Además, la mayoría de los medios anuncia varias veces por día y con distintos grados de originalidad 
quiénes son los responsables de cada emisora y si se trata de una empresa, de una persona o de un colectivo sin fines de lucro. Aunque todavía lo más importante es materia del futuro.

La transición en la oferta audiovisual será un proceso complejo y largo. El nuevo marco legal establece condiciones que obligarán, por ejemplo, a incrementar la producción propia de contenidos de las señales. Este es un elemento central, sobre todo si se parte de situaciones como las que demuestra el séptimo Informe de contenidos de TV abierta ${ }^{17}$, que pone de manifiesto el centralismo del sistema y la alta penetración de los contenidos emitidos por los canales de cabecera propiedad del Grupo Clarín y Telefónica, y destaca que del total de horas emitidas en los canales de televisión, el 86\% es la retrasmisión de producciones de Canal $11(44 \%)$ y de Canal 13 (42\%). Los nuevos mínimos de producción local y propia ${ }^{18}$ habilitan a esperar más contenidos locales, regionales y federales aunque los resultados no se perciben aún de modo concreto.

Los nuevos contenidos que podrían generarse se completan, además, con la exhibición de varios filmes de producción nacional de los muchos que se realizan en nuestro país con dinero del INCAA, a fin de establecer nuevas ventanas de distribución para esas producciones y garantizar mejores condiciones de acceso de los ciudadanos para un cine que se produce en el país pero que no se mira (masivamente). También se crean las condiciones para que los canales y productoras (definidas en sentido amplio, con y sin fines de lucro) realicen telefilmes y productos de ficción, muy relevantes para el desarrollo de la cultura local, para la construcción de la identidad y para la generación de empleos en esta área. De todos modos, como avances, este resumen muestra que lo que se puede percibir en la pantalla es más bien pobre.

\section{Un camino paralelo: la televisión digital terrestre (TDT)}

La TDT, como una plataforma de transmisión de señales de televisión digital en la Argentina, comenzó a desarrollarse en 2009 por iniciativa del gobierno nacional luego de la adopción de la norma japonesa ISBT (Rodríguez Miranda, 2011) y se encuentra en pleno proceso de desarrollo. Se han completado en julio de 2011 dos de las cuatro fases planificadas, con lo que se logró cubrir el territorio en el que habita el 65\% de los ciudadanos argentinos, unos 9,5 millones de hogares. De

\footnotetext{
17 Realizado y distribuido por la Autoridad Federal de Servicios de Comunicación Audiovisual.

18 Según cada tipo de medio y zona de cobertura.
} 
acuerdo con el último dato registrado, para lograr que más del 82\% de la población nacional disfrute de este servicio, se instalaron 71 estaciones digitales de transmisión (EDT) en diferentes puntos del país que emiten la señal de la TDA. Este plan ya entregó más de 1.200.000 equipos receptores terrestres de manera gratuita a hogares, establecimientos y organizaciones sociales. Como complemento, también se han instalado más de 1.800 antenas de TV digital satelital (TDS) en parajes rurales y más de 11.000 en escuelas rurales y de frontera, para que todos los argentinos tengan igualdad de oportunidades en el acceso a la TDA ${ }^{19}$. Ahora bien, el total de personas que pueden ver la TDT es sensiblemente menor. Si bien no hay datos oficiales (ni privados ni públicos), para Martín Becerra, investigador de CONICET y profesor de la Universidad Nacional de Quilmes, "se estima que poco menos del $10 \%$ de quienes están cubiertos por la antenas de TDT acceden a sus señales, por la cantidad de decodificadores distribuidos a la fecha" que cuentan con el equipamiento necesario para ello: un televisor de nueva generación con recepción digital o un decodificador para adaptar un equipo analógico (Marino, 2012a).

La inversión que lleva adelante el Estado desde el Ministerio de Planificación Federal, Inversión Pública y Servicios, se implementa en una Argentina de 14,2 millones de hogares ${ }^{20}$, en la que el 97,8\% tiene al menos un receptor de TV y hay más de 7,5 millones de abonados a televisión de pago (Marino, 2012b). En ese contexto, el gobierno llevó adelante un plan para entregar 1.180.000 decodificadores a personas de sectores sociales vulnerables. Entre los beneficiados están quienes cobran la jubilación mínima y la asignación universal por hijo (AUH). Hacia fines de 2013 los datos oficiales sostienen que el plan se completó en un 80,3\% ya que se entregaron 948.120 decodificadores ${ }^{21}$.

\section{Conclusiones}

El nuevo escenario normativo, con objetivos democratizadores y compatible con la defensa del derecho a la información, avanza sin embargo con una serie de cuestiones pendientes, entre las que se destacan elementos propios de la aplicación sesgada de la norma o bien cuestiones que no podían (o debían) ser resueltas en la ley. En principio, resulta urgente un debate sobre la aprobación del plan técnico de frecuencias, que permitirá conocer, entre otros aspectos, la cantidad de emisoras

\footnotetext{
19 El enlace al mapa de cobertura es http://www.tda.gob.ar/cobertura.html

20 www.censo2010.indec.gov.ar/

21 http://estadisticas.tda.gob.ar/
} 
que el espectro radioeléctrico nacional soporta y así tener en claro cuántas corresponden al tercio reservado como objetivo democratizador.

El plexo normativo definido desde el Estado para el sector de la radiodifusión desde 2002 a 2009 benefició al sector privado y profundizó la estructura de concentración, los aplicados en el sector del cine mejoraron las políticas de apoyo a la producción y a la exhibición para las películas nacionales. Desde 2009 en adelante la regulación cambia sus objetivos y establece tanto una serie de límites a la concentración de la propiedad como un intento de fomentar la diversidad de los contenidos en el sector de la radiodifusión. La política de cine se mantiene y sus resultados en materia de producción de contenidos son satisfactorios, no así los de distribución y exhibición. En ese marco debe incluirse además el avance tecnológico y la novedad de la digitalización.

Algunos aspectos que resultan de esta política pueden evaluarse como dilemas a resolver; en primer lugar, el recorrido paralelo —en términos matemáticos, son dos líneas rectas que nunca se tocan-de la política de TDT y la ley de medios; en segundo lugar, que el organismo de aplicación sea el Ministerio de Planificación, sin vínculo con la AFSCA; y en tercer lugar, los resultados de esta gestión dan cuenta de la inexistencia de una planificación integral para el sector audiovisual que integre la política del cine del INCAA con el resto de los organismos.

Con la Ley de Servicios de Comunicación Audiovisual en plena vigencia existen temas pendientes que no dependen de la aplicación de la norma y son cuestiones centrales. Entre ellos, se destaca la incomprensible situación de que su aplicación y la implementación de la política de TDT vayan por carriles paralelos, lo cual resulta incongruente con los mejores objetivos de las directrices democratizadoras de la actual gestión gubernamental. Por otro lado, el modelo de financiación de las organizaciones que cuentan ahora con derechos reconocidos pero a la vez con obligaciones por cumplir (en materia de producción de contenidos propios, infraestructura y cuestiones de funcionamiento) es uno de los desafíos centrales que enfrentan los medios comunitarios, a quienes se comprende como el emergente comunicacional más importante desde el regreso de la democracia en la Argentina, muchos de los cuales han dado sobradas muestras de responder a proyectos integrales y ambiciosos en escenarios negativos y de exclusión.

Si bien la diversidad de la oferta no está garantizada por la ley, pensar en un sistema audiovisual con mayor variedad no es una utopía sino un desafío a resolver. Porque los niveles exigidos demandarán mucha inversión en la producción, sin 
que quede claro aún qué tipo de contenidos podrán generarse, cuáles serán los estándares de calidad y sobre todo, cuáles las fuentes de financiamiento. Responder a esta pregunta es urgente. Pensar mecanismos de promoción es necesario. El hecho de prestar atención a los cambios será clave para identificar si se modifica —como se espera- la oferta cultural para ver esa ley en las pantallas.

La política diseñada a mediados de la larga década neoliberal para el cine generó una serie de efectos que permite caracterizar al sector como el de la paradoja de las políticas públicas del audiovisual. Logró transformar las condiciones de producción de filmes nacionales y ampliar la diversidad de la oferta, aunque no tuvo efectos en las instancias de distribución y exhibición, que mantuvieron y profundizaron una estructura concentrada. Su continuidad luego del estallido de la crisis de 2001 implicó la consolidación de política pública adoptada en un momento único y particular como una política de Estado consolidada, otro rasgo peculiar.

La presencia de un conjunto de acciones que garantizan la existencia de producción local de cine, la relevancia de su permanencia en el tiempo y su impronta, generan legitimidad en un modelo sobre el que mucho se habla pero del que poco se discute en profundidad. La necesidad de repensar y reorientar sus directrices, sin que esto afecte la garantía de su continuidad, resulta muy clara.

Mientras tanto, una serie de interrogantes se instalan, sin respuestas posibles todavía: ¿cuál es el escenario futuro del espacio audiovisual argentino? ¿cuáles son las posibilidades reales de desarrollo de un proyecto sustentable más allá del financiamiento estatal o de los grandes jugadores del mercado? ¿cómo pensar un proyecto inclusivo en materia de acceso a los productos generados con dinero público y cuánto se transformó el sistema para garantizar la participación ciudadana? Y eso que el futuro llegó, hace rato.

\section{Referencias}

Getino, O. (2005). Cine argentino: entre lo posible y lo deseable. Buenos Aires: Ediciones CICCUS; Instituto Nacional de Cine y Artes Audiovisuales (INCAA).

Lacunza, S. \& Marino, S. (2013). "Una encrucijada para dos: Clarín y el gobierno". En: Ámbito Financiero, $1^{\circ}$ de noviembre, suplemento "Viernes". Disponible en internet: http://www.ambito. com/suplementos/viernes/noticia_suplemento.asp?ID=714083\&Seccion=Secciones+Especiales

Marino, S. (2012a). Políticas de comunicación del sector audiovisual: las paradojas de modelos divergentes con resultados congruentes: los casos de la televisión por cable y el cine en Argentina entre 1989-2007 [tesis doctoral]. Buenos Aires, [defendida el 18 de abril de 2013], Universidad de Buenos Aires.

Marino, S. (2012b). CFK: "Hoy estamos cubriendo al 65\% de la población argentina con la TV digital abierta. En: Chequeado.com [en línea]. Buenos Aires: Fundación La Voz Pública, 22 de febrero. 
Disponible en internet: http://chequeado.com/ultimas-noticias/940-cfk-hoy-estamos-cubriendoal-65-de-la-poblacion-argentina-con-la-tv-digital-abierta-y-gratuita-para-todos.html.

Marino, S.; Mastrini, G. \& Becerra, M. (2010). El proceso de regulación democrática de la comunicación en Argentina. Oficios Terrestres. La Plata: Universidad Nacional de La Plata. Facultad de Periodismo y Comunicación Social, 16 (25), p. 11-24. Disponible en internet: http:// www.perio.unlp.edu.ar/oficios/documentos/pdfs/oficios_25.pdf

Mastrini, G. \& Loreti, D. (2013). "Sobre el fallo de la cámara en el caso Clarín". En: Página 12, Buenos Aires, 14 de mayo. Disponible en internet: http://www.pagina12.com.ar/diario/ elpais/1-219941-2013-05-14.html

Rodríguez Miranda, C. (2011). Políticas públicas para la televisión digital terrestre: el proceso de adopción del sistema técnico ISDB-Tb: casos Argentina y Chile [tesis]. Maestría en industrias culturales, políticas y gestión, Universidad Nacional de Quilmes.

Sahores, E. (2013). El cine argentino en la era del "Nunca menos": nuevos procesos de concentración y extranjerización durante el período 2009-2012 [tesina]. Buenos Aires. Licenciatura en Ciencias de la Comunicación, Facultad de Ciencias Sociales, Universidad de Buenos Aires.

\section{Contacto}

\section{Santiago Marino}

sgomarino@gmail.com 